\title{
Comparaison des performances de croissance en station de pisciculture d'une souche sauvage et d'une souche domestique du tilapia du Nil (Oreochromis niloticus L.) au Sud-Kivu, RD Congo
}

\author{
NIHOREYE Fakage John ${ }^{1 *}$, NYONGOMBE Utshudienyema Nathan², ALUNGA Lufungula Georges ${ }^{3,4}$ \\ et UMBA di M'Balu Joachim ${ }^{5}$ \\ 1Département de Biologie, Institut Supérieur Pédagogique de Bukavu. BP 854 Bukavu, RD Congo \\ ${ }^{2}$ Faculté des Sciences Agronomiques, Département de Zootechnie, Université Pédagogique Nationale. BP 8815 \\ Kinshasa, RD Congo \\ 3Centre de Recherches Universitaires du Kivu, Institut Supérieur Pédagogique de Bukavu. BP 854 Bukavu, RD \\ Congo \\ ${ }^{4}$ Unité d'Enseignement et de Recherche en Hydrobiologie Appliquée, Institut Supérieur Pédagogique de Bukavu. BP \\ 854 Bukavu, RD Congo \\ ${ }^{5}$ Faculté de Médecine Vétérinaire, Université Pédagogique Nationale. BP 8815 Kinshasa, RD Congo \\ *Auteur correspondant, E-mail : jakage@hotmail.com
}

Original submitted in on $16^{\text {th }}$ May 2019. Published online at www.m.elewa.org/journals/ on $31^{\text {st }}$ August 2019 https://dx.doi.org/10.4314/jab.v140i1.4

\section{RÉSUMÉ}

Objectif : Deux souches du tilapia du Nil Oreochromis niloticus, trouvées dans la région du Sud-Kivu en RD Congo, dont une récemment prélevée du milieu naturel (souche Lac Kivu) et une autre ayant un long passé de domestication (souche Nyakabera) ont été comparées en station de pisciculture en vue de déterminer laquelle avait les meilleures performances de croissance.

Méthodologie et résultats : Après 183 jours de maintien dans des conditions zootechniques similaires, les poissons de taille marchande dans les deux souches étaient dans une bonne condition $(K>1)$, toutefois avec une supériorité $(p<0,05)$ de la souche Lac Kivu $(K=1,80 \pm 0,02)$ par rapport à la souche Nyakabera $(\mathrm{K}=1,71 \pm 0,01)$. Les poissons pesant $200 \mathrm{~g}$ et plus représentaient $85 \%$ de la récolte chez la souche Lac Kivu contre $70 \%$ chez la souche Nyakabera. Le poids moyen final des géniteurs initiaux était de 285,4 $\pm 3,6$ $\mathrm{g}$ et $199,5 \pm 3,1 \mathrm{~g}$, respectivement pour la souche Lac Kivu et la souche Nyakabera. Le gain de poids individuel était de $278,0 \pm 4,9 \mathrm{~g}$ et $187,1 \pm 5,1 \mathrm{~g}$, la croissance journalière individuelle de 1,52 $\pm 0,16 \mathrm{~g} / \mathrm{jour}$ et $1,02 \pm 0,19 \mathrm{~g} / \mathrm{jour}$, le taux de croissance spécifique de $1,99 \pm 0,03 \% /$ jour et $1,52 \pm 0,03 \% /$ jour, respectivement pour la souche Lac Kivu et la souche Nyakabera. Pour ces quatre derniers paramètres, les différences entre les deux souches étaient significatives $(p<0,05)$ en faveur de la souche Lac Kivu qui a ainsi manifesté les meilleures performances de croissance.

Conclusion et application des résultats: Les performances de croissance des souches Lac Kivu et Nyakabera d'Oreochromis niloticus sont liées à leur origine ainsi que leur histoire de domestications respectives. La contre-performance de la souche Nyakabera serait liée aux effets pervers du faible effectif du stock de géniteurs à partir duquel elle a été fondée à l'origine. Les pisciculteurs du Sud-Kivu et des 
régions avoisinantes peuvent désormais utiliser préférentiellement les alevins produits à partir de géniteurs du tilapia du Nil du lac Kivu pour améliorer les rendements de leurs exploitations.

Mots-clés : facteur de condition, lac Kivu, conditions zootechniques, taux de croissance spécifique, taux de survie.

\begin{abstract}
Objective : Two strains of Nile tilapia Oreochromis niloticus, found in the South Kivu region of DR Congo, one recently taken from the natural environment (Lac Kivu strain) and another with a long history of domestication (Nyakabera strain) were compared in fish farm to determine which had the best growth performance.

Methodology and results : After 183 days of maintenance in similar zootechnical conditions, the commercial size fish in both strains were in good condition $(K>1)$, but with a superiority $(p<0.05)$ of the Lac Kivu strain $(K=1.80 \pm 0.02)$ relative to the Nyakabera strain $(K=1.71 \pm 0.01)$. At the harvest, fish weighing $200 \mathrm{~g}$ or more accounted for $85 \%$ in the Lac Kivu strain compared to $70 \%$ in the Nyakabera strain. The final average weight of the starting broodfish was $285.4 \pm 3.6 \mathrm{~g}$ and $199.5 \pm 3.1 \mathrm{~g}$ for the Lac Kivu strain and the Nyakabera strain, respectively. The individual weight gain, individual daily growth, and specific growth rate were respectively $278.0 \pm 4.9 \mathrm{~g} ; 1.52 \pm 0.16 \mathrm{~g} /$ day and $1.99 \pm 0.03 \% /$ day for Lac Kivu strain and $187.1 \pm 5.1 \mathrm{~g}$; $1.02 \pm 0.19 \mathrm{~g} /$ day and $1.52 \pm 0.03 \% /$ day for the Nyakabera strain. For these last four parameters, the differences between the two strains were significant $(p<0.05)$ in favor of the Lac Kivu strain, which thus showed the best growth performance.

Conclusion and application of results: The growth performance of the Lac Kivu and Nyakabera strains of Oreochromis niloticus are related to their respective origin and domestication history. The Under performance of the Nyakabera strain would be related to the adverse effects of the low stock size of the parent stock from which it was originally based. Fish farmers in South Kivu and neighboring regions can now preferentially use fry produced from Lake Kivu Nile tilapia broodstock to improve the yields of their farms.
\end{abstract}

Keywords: condition factor, Lake Kivu, zootechnical conditions, specific growth rate, survival rate.

\section{INTRODUCTION}

Le tilapia du Nil, Oreochromis niloticus (Linnaeus, 1758) est une espèce largement élevée car il croît et se reproduit dans un large éventail de conditions environnementales et tolère le stress induit par les manipulations (Tsadik \& Bart, 2007). Cependant, dans beaucoup de systèmes d'élevage l'efficacité de la reproduction chez les tilapias a des conséquences indésirables. Elle conduit à la réduction des taux de croissance au début de la maturité sexuelle et à une reproduction précoce et excessive, le tout résultant en une production de nombreux individus de petite taille (Lazard \& Legendre, 1996; Chakraborty et al., 2011). En effet, dans ce contexte, au sein de la population en élevage, les mâles, et spécialement les femelles, détournent l'énergie qui devrait être utilisée pour la croissance somatique dans la production des gamètes et les interactions comportementales. En outre, la compétition avec les recrues dans des environnements confinés réduit davantage la croissance des poissons élevés et peut avoir pour résultat que 30 à $50 \%$ de biomasse récoltée est constituée de petits poissons non commercialisables (Mair et al., 1995). Ce problème de prolificité se pose avec acuité dans l'élevage d'O. niloticus en sexes mélangés en étangs en terre, plus que dans les cages, les hapas et les bassins où les conditions de reproduction ne sont pas autant favorables. De nombreuses solutions à ce problème ont été proposées, notamment le sexage manuel et la séparation des sexes, la culture en cages, l'utilisation contrôlée d'espèces prédatrices, la production d'hybrides monosexes et l'inversion du sexe par voie hormonale, thermique ou génétique (Mair \& Little, 1991; Lazard, 2009). Les techniques 
de sexage visent l'élevage des populations monosexes mâles, car les mâles ont chez 0 . niloticus une croissance en moyenne de $50 \%$ supérieure aux femelles (Lazard, 2009). Cependant, toutes les techniques susmentionnées comportent d'importants inconvénients, particulièrement pour une application dans les pays en développement (Mair et al., 1995; Akongyuure et al., 2015) et spécialement en contexte rural où les pisciculteurs travaillent dans des conditions socioéconomiques plus contraignantes. Par ailleurs, la problématique du taux de croissance chez 0 . niloticus se pose aussi lorsqu'il faut examiner le comportement des souches d'élevage comparativement aux souches sauvages. II est établi que le tilapia du Nil souffre de la détérioration génétique en conditions d'élevage en raison de l'introgression généralisée de gènes d'autres espèces de tilapia sauvages moins désirables et d'une possible dépression de consanguinité (Abdel-Tawwab, 2004). Bon nombre des stocks de cette espèce mis en culture ont été créés à partir d'un nombre limité de poissons capturés à l'état sauvage entre les années 1960 et 1970 et ont été par la suite redistribués à travers le monde (Khater \& Smitherman, 1988; Pullin \& Capili, 1988). Certains de ces stocks ont une histoire assez compliquée avec des transferts successifs, multipliant les risques d'apparition de goulots d'étranglement (Rognon \& Guyomard, 1996; Vrijenhoek, 1998). L'effet des petites populations fondatrices conjugué à celui de la nature prolifique du tilapia offre la possibilité d'une dépression de consanguinité. II a été largement rapporté que la consanguinité chez les poissons entraîne une baisse significative de leurs performances, résultant d'une perte de diversité génétique qui affecte négativement le taux de croissance, le taux de survie et les activités de reproduction (Bondari et Dunham, 1987; Gjerde, 1988 ; Galman et al., 1988; Leberg, 1990; Agnèse et al., 1995; Hussain, 2004). Dans de telles circonstances, les poissons n'arrivent pas à atteindre des tailles marchandes dans un cycle de production (Liti et al., 2005). Eknath et al. (1993), évaluant les performances de croissance de huit différentes souches d'O. niloticus dont quatre récemment collectées du milieu naturel et quatre ayant une longue histoire de domestication, avaient constaté que les trois souches les plus performantes étaient parmi les plus récemment collectées. La même observation avait été faite par Brummet et al. (2004) qui avaient trouvé que les alevins d'O. niloticus issus de géniteurs récemment capturés du milieu naturel manifestent une croissance remarquablement plus rapide que ceux des souches domestiquées depuis plusieurs années, tant en situation de station de pisciculture qu'en celle de la ferme $(39 \%$ et $42 \%$ respectivement). Dans la province du Sud-Kivu (en RD Congo), le tilapia du Nil est largement utilisé en pisciculture mais il y existe aussi à l'état sauvage dans les principaux hydrosystèmes tels que les lacs Kivu et Tanganyika et la rivière Ruzizi (Snoeks et al., 1997). Ici, les souches d'élevage sont habituellement importées (donc d'origine allochtone) alors que les populations trouvées dans les trois milieux naturels proviennent, selon Snoeks et al. (1997), d'une espèce autochtone au lac Kivu. Dans cette Province, la pisciculture mobilise plusieurs petits exploitants ruraux qui élèvent principalement 0 . niloticus dans des étangs en terre en systèmes de sexes mélangés. La souche majoritairement utilisée dans ce contexte (dite souche Nyakabera) est d'une origine lointaine et a une longue histoire de domestication (Nihoreye et al., soumis). Une enquête menée récemment auprès des pisciculteurs dans plusieurs contrées de la Province a noté des rendements médiocres de façon chronique, en termes de quantités de poissons récoltés et de leur poids marchand à l'issue d'un cycle de production. Cette contre-performance a été mise en lien avec des pratiques piscicoles inadéquates (étangs peu ou pas fertilisés, nourrissage non pratiqué) mais aussi avec l'utilisation des alevins dont la croissance est jugée non satisfaisante (Nihoreye et al., soumis). Les performances zootechniques des souches d'O. niloticus utilisées en pisciculture au Sud-Kivu n'ont pas encore été scientifiquement évaluées et comparées à celles des populations naturelles. Et pourtant, selon Bentsen et al. (1998) et Amoussou et al. (2017), une telle approche constitue une étape préalable de tout processus 
de mise en œuvre d'un programme de sélection visant à optimiser les performances zootechniques des souches locales. A ce propos, l'une des souches sauvages pourrait constituer une alternative par rapport aux insuffisances constatées chez la souche en cours d'utilisation. Certaines études (Reisenbichler, 1988; McGinnity et al., 2003; Araki \& Schmid, 2010) ont montré que les poissons d'élevage issus des populations

\section{MATÉRIEL ET MÉTHODES}

Site d'étude : Cette étude a été conduite de juillet 2016 à janvier 2017 à la station piscicole étatique de Nyakabera, située en périphérie de la ville de Bukavu, en Province du Sud-Kivu, République Démocratique du Congo. La station piscicole de Nyakabera est à $02^{\circ} 28^{\prime}$ de latitude Sud, $28^{\circ} 49^{\prime}$ de longitude Est et $1553 \mathrm{~m}$ d'altitude. Le site compte actuellement une trentaine d'étangs de dérivation en terre (totalisant une centaine d'ares) dédiés essentiellement à la production des alevins.

Souches de poisons utilisées : La présente étude a porté sur deux souches du tilapia du Nil Oreochromis niloticus, respectivement nommées "Nyakabera » et "lac Kivu», élevées à la station piscicole de Nyakabera. La souche d'O. niloticus dite "lac Kivu» existe à la station piscicole de Nyakabera depuis avril 2014. Elle a été introduite à Nyakabera depuis le lac Kivu dans le bassin de Bukavu, dans le cadre d'un essai d'acclimatation. 0 . niloticus est la seule espèce autochtone sur les 4 espèces de tilapia signalées au lac Kivu (Snoeks et al., 1997). Les poissons de cette souche ayant fait l'objet de ce travail appartenaient à la quatrième génération depuis son introduction à la station piscicole de Nyakabera. Une introduction qui avait été réalisée avec 340 jeunes géniteurs de 30 à 60 g, dont 160 mâles et 180 femelles. La souche domestique d'O. niloticus utilisée dans cette étude a été introduite en 2005 à Nyakabera depuis la station piscicole de Rwasave, une station de l'Université Nationale du Rwanda à Butare. Elle est à l'origine un mélange de souches égyptienne et ivoirienne, ellesmêmes amenées à Rwasave depuis une station de pisciculture de l'Université d'Auburn (USA) entre 1984 et 1985. II s'agirait de la souche «Rwanda-USA 1984 », selon FishBase, dont le stock fondateur était de 22 femelles et 18 mâles (Pullin, 1988). Depuis son arrivée à la station de Nyakabera, cette souche est disséminée dans plusieurs contrées des provinces de l'Est de la RD Congo à travers des programmes locales ont tendance à obtenir de meilleurs résultats sur la plupart des critères que ceux transférés. C'est dans ce cadre que la présente étude se propose d'évaluer et de comparer en conditions d'élevage quelques performances de croissance de deux souches d'O. niloticus présentes dans la région du Sud-Kivu, dont une souche domestique allochtone (dite Nyakabera) et une souche sauvage autochtone (dite Lac Kivu).

d'assistance aux paysans pisciculteurs implémentés notamment par les organisations humanitaires (Nihoreye et al., 2009).

Dispositif expérimental : L'expérimentation a été conduite dans six étangs de dérivation en terre de 2 ares avec une profondeur moyenne de $1 \mathrm{~m}$ chacun. Les étangs étaient alimentés en eau par un ruisseau, les tuyaux à l'entrée étant munis d'un treillis métallique à mailles fines pour limiter l'invasion par des poissons étrangers indésirables. Les alevins en sexes mélangés de deux lots de poissons ont été affectés au hasard dans les étangs ; ainsi l'expérimentation a été réalisée en triplicat dont trois étangs étaient chargés avec la souche Lac Kivu et trois autres avec la souche Nyakabera. Le taux de mise en charge uniforme de 1,25 poisson $/ \mathrm{m}^{2}$ a été appliqué pour tous les étangs. Les alevins utilisés avaient un poids moyen ( \pm erreur standard) initial de 6,1 $\pm 0,6 \mathrm{~g}$ et de 11,1 $\pm 0,6 \mathrm{~g}$, respectivement pour Lac Kivu et Nyakabera. II est à noter que, d'après Palada-de Vera \& Eknath (1993) et Jayaprakas et al. (1988), l'écart dans le poids initial des alevins a un effet limité sur leurs performances de croissance ultérieures. Les performances de croissance étant traduites essentiellement sous forme de taux de croissance spécifique (\%/j), c'est l'écart de poids par rapport à la durée du cycle d'élevage qui compte plus que l'écart absolu entre le poids final et le poids initial. Afin de réguler le $\mathrm{pH}$ de l'eau de l'étang et la chimie de l'eau en général et éliminer les parasites tels que les sangsues et les larves des grenouilles, avant le stockage des poissons, les étangs ont été drainés et chaulés avec de la chaux agricole à raison de 0,25 $\mathrm{kg} / \mathrm{m}^{2}$ (Engle \& Neira, 2005). La fertilisation était partout réalisée avec des feuilles de Tithonia $s p$ placées dans une compostière de $4 \mathrm{~m}^{2}$ aménagée dans un coin de l'étang. Les compostières étaient rechargées une fois par semaine. Les poissons étaient nourris avec un aliment constitué d'un mélange de drèche de brasserie $(50 \%)$, de tourteau palmiste $(30 \%)$ et de farine de fretin 
(20\%). La distribution de l'aliment se faisait manuellement deux fois par jour, le matin entre 9 heures et 10 heures et le soir entre 15 heures et 16 heures. Le taux journalier de nourrissage appliqué était de $5 \%$ de la biomasse estimée des poissons (à partir d'un échantillonnage mensuel incluant toutes les classes de tailles).

Détermination des paramètres de croissance : Un échantillon de 50 poissons parmi les géniteurs était prélevé mensuellement dans chaque étang à l'aide d'une senne. Ces poissons vifs étaient anesthésiés au phénoxyéthanol $0,02 \%$ avant la pesée individuelle à l'aide d'une balance électronique d'une précision de $1 \mathrm{~g}$ et la mesure de la longueur à $1 \mathrm{~mm}$ près à l'aide d'un ichthyomètre. A chaque occasion, les poissons étaient replacés dans leurs étangs respectifs aussitôt après les mensurations. Après 183 jours d'élevage, tous les étangs avaient été récoltés par vidange totale. Les poissons initialement mis en charge avaient été séparés des juvéniles et des alevins, puis comptés et pesés en lots par souche et par étang. Pour chaque lot, un échantillon de 50 poissons était prélevé au hasard pour être mesurés et pesés individuellement. Le nombre et le poids de la progéniture n'ont pas été inclus dans les calculs. Les paramètres de croissance tels que le gain de poids moyen (GPM), le gain de poids journalier (GPJ), le taux de croissance spécifique (TCS), le facteur de condition (K) ainsi que le taux de survie (TS) ont été calculés suivant les équations ciaprès (Pechsiri \& Yakupitiyage, 2005 ; Ridha, 2006; Jiwyam, 2011) :

GPM $(\mathrm{g})=$ poids moyen final $(\mathrm{g})$ - poids moyen initial (g).

GPJ $(\mathrm{g} / \mathrm{jour})=$ [poids moyen final $(\mathrm{g})$ - poids moyen initial (g)]/durée élevage (jours).

TCS (\%/jour) $=100[(\ln \mathrm{Pf}-\ln \mathrm{Pi}) / \mathrm{t}] ;$ où $: \ln =$ logarithme naturel; $\mathrm{Pi}=$ poids initial $(\mathrm{g}) ; \mathrm{Pf}=$ poids final (g) ; $\mathrm{t}=$ durée élevage en jours.

$\mathrm{K}=\mathrm{PT} / \mathrm{LT}^{3} \times 100$; où: $\mathrm{PT}=$ poids total $(\mathrm{g}) ; \mathrm{LT}=$ longueur totale $(\mathrm{cm})$.

TS $(\%)=$ (nombre de poissons récoltés/nombre de poissons à la mise en charge) x100.

Analyses statistiques des données: Les différents calculs et les graphiques ont été réalisés grâce au logiciel Microsoft Excel 2010. La signification des différences entre les moyennes pour les différents paramètres étudiés a été appréciée au seuil de $5 \%$ par le test non paramétrique $U$ de Mann-Withney, à l'aide du logiciel PAST version 3.18 (Hammer et al., 2001).

\section{RÉSULTATS}

Les poids moyens des poissons enregistrés au début et à la fin de l'expérimentation ainsi que les performances de croissance de deux souches d'Oreochromis niloticus examinées, en termes de gain de poids moyen, de gain de poids journalier, de taux de croissance spécifique, de facteur de condition $\mathrm{K}$ et de taux de survie, sont présentés dans le tableau 1.

Tableau 1: Paramètres de croissance des souches Lac Kivu et Nyakabera d'Oreochromis niloticus (moyenne \pm erreur standard)

\begin{tabular}{l|c|c|c}
\hline \multirow{2}{*}{ Paramètres de croissance } & \multicolumn{2}{|c|}{ Souches } & $\mathbf{p}$ \\
\cline { 2 - 4 } & Lac Kivu & Nyakabera & 0,0182 \\
Poids moyen initial (g) & $6,1 \pm 0,6$ & $11,1 \pm 0,6$ & 0,0109 \\
Poids moyen final (g) & $285,4 \pm 3,6$ & $199,5 \pm 3,1$ & 0,0100 \\
Gain de poids moyen (g) & $278,0 \pm 4,9$ & $187,1 \pm 5,1$ & 0,0256 \\
Gain de poids journalier (g/jour) & $1,52 \pm 0,16$ & $1,02 \pm 0,19$ & 0,0199 \\
Taux de croissance spécifique (\%/jour) & $1,99 \pm 0,03$ & $1,52 \pm 0,03$ & 0,0286 \\
Facteur de condition K & $1,80 \pm 0,02$ & $1,71 \pm 0,01$ & 0,0613 \\
Taux de survie (\%) & $86,4 \pm 1,5$ & $89,8 \pm 1,1$ & \\
\hline
\end{tabular}

La souche Lac Kivu s'est montrée plus performante que la souche Nyakabera pour tous les paramètres étudiés, excepté le taux de survie pour lequel la différence observée n'est pas significative. Au démarrage de l'expérimentation, les alevins de la souche Nyakabera avaient un poids moyen de $11,1 \pm 0,6 \mathrm{~g}$, supérieur $(p<0,05)$ à celui des alevins de la souche Lac Kivu
$(6,1 \pm 0,6 \mathrm{~g})$. A la récolte, le poids individuel final atteint par les poissons de la souche Lac Kivu $(285,4 \pm 3.6 \mathrm{~g})$ est en moyenne de $43,1 \%$ supérieur à celui des poissons de la souche Nyakabera qui est de 199,5 \pm 3.1 g. Cette tendance s'observe également pour le gain de poids moyen, le gain de poids journalier, le taux de croissance spécifique et le facteur de condition pour 
lesquels la souche Nyakabera a affiché respectivement une infériorité de $32,7 \%, 32,9 \%, 23,6 \%$ et $5 \%$ par rapport à la souche Lac Kivu. Les deux souches d'O. niloticus se sont caractérisées par une tendance similaire de la courbe de croissance (Figure 1).

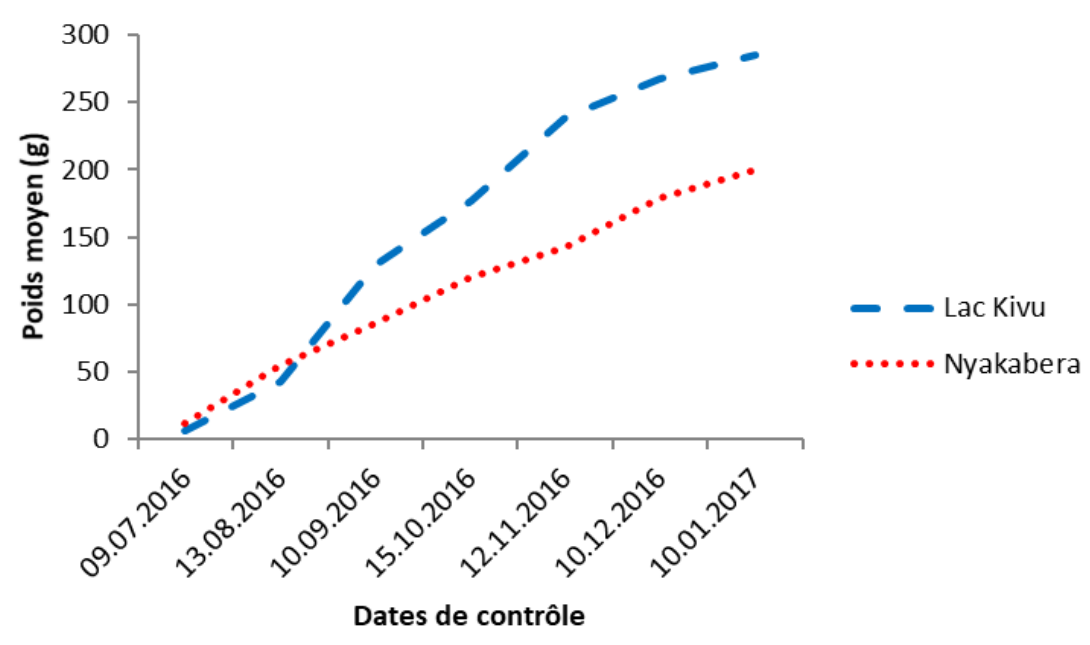

Figure 1. Evolution comparée des poids moyens des poisons au cours de la période d'élevage pour les souches Lac Kivu et Nyakabera d'Oreochromis niloticus

Les résultats ont révélé une augmentation assez constante du poids moyen depuis la mise en charge (juillet 2016) jusqu'à la récolte (janvier 2017). Ainsi, pour la souche Nyakabera le poids moyen était de 11,1 $\mathrm{g}$ à l'ensemencement, $54,6 \mathrm{~g}$ le premier mois, $86,3 \mathrm{~g}$ le deuxième mois, $119,7 \mathrm{~g}$ le troisième mois, $142,8 \mathrm{~g}$ le quatrième mois, $179 \mathrm{~g}$ le cinquième mois et $199,5 \mathrm{~g}$ à la récolte finale. Aux mêmes périodes, il a été noté respectivement $6,1 \mathrm{~g} ; 42,3 \mathrm{~g} ; 129,5 \mathrm{~g} ; 176,1 \mathrm{~g} ; 238,7$ $\mathrm{g} ; 267 \mathrm{~g}$ et 285,5 g pour la souche Lac Kivu. Cependant, 4 semaines après la mise en charge des étangs expérimentaux la courbe de croissance de la souche Lac Kivu se sépare de celle de la souche Nyakabera et la surclasse ensuite, de manière continue, dès ce moment jusqu'à la récolte. Les résultats relatifs au poids moyen enregistrés à la récolte ont révélé que dans les deux souches le plus grand nombre de poissons étaient dans la classe de 200 à $250 \mathrm{~g}$, suivi de celle de moins de $200 \mathrm{~g}$ tandis que les poissons de plus de $250 \mathrm{~g}$ étaient partout minoritaires (Figure 2).
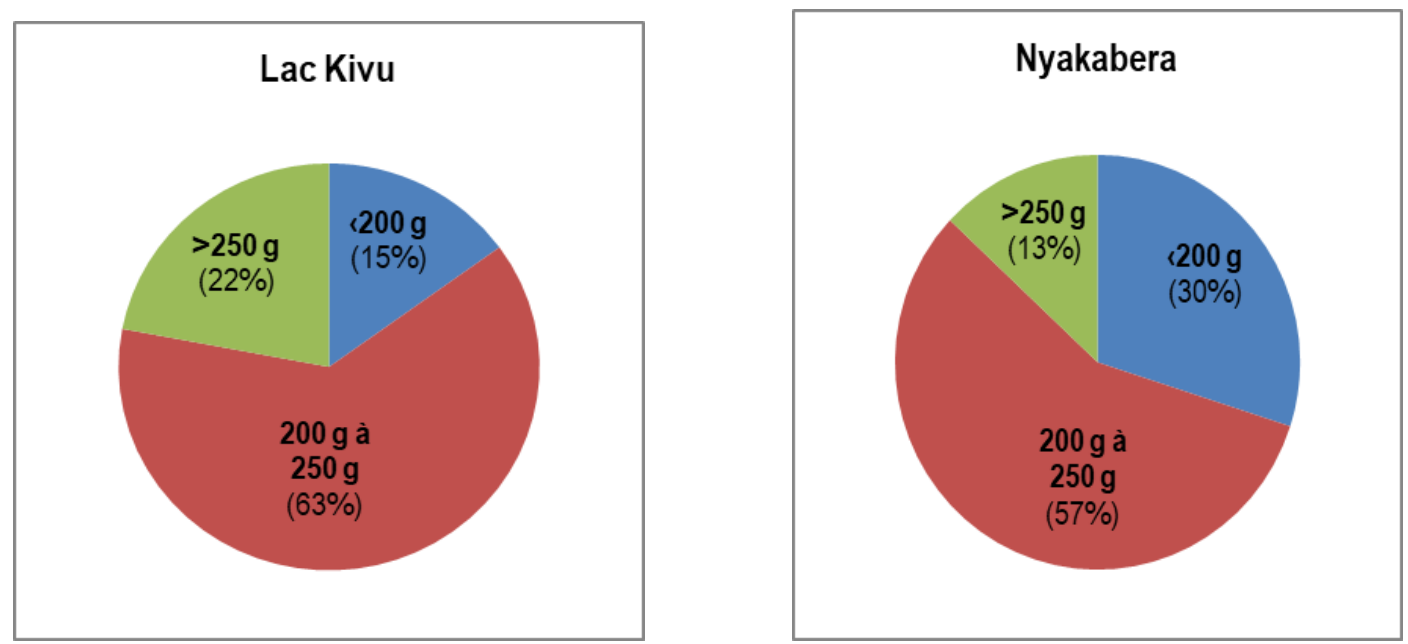

Figure 2 : Distribution des classes de poids à la récolte chez les souches Lac Kivu et Nyakabera d'Oreochromis niloticus 
En termes de taille marchande, les proportions observées chez la souche Lac Kivu pour les classes 200 à $250 \mathrm{~g}$ et plus de $250 \mathrm{~g}$ sont chaque fois supérieures à celles de la souche Nyakabera, soit

\section{DISCUSSION}

L'objectif de cette étude était de comparer le comportement de deux souches du tilapia du Nil Oreochromis niloticus dont l'une (la souche Nyakabera) ayant une longue histoire de domestication et l'autre (la souche Lac Kivu), autochtone de la région du Sud-Kivu, qui a été récemment introduite en pisciculture en provenance du milieu naturel (le lac Kivu). A cet effet, les deux souches ont été soumises à des conditions zootechniques supposées similaires dans le but de mettre en évidence un éventuel impact de leurs différences variétales sur les performances de croissance. La présente étude a montré que la différence de croissance observée entre les deux souches n'est pas liée à l'alimentation étant donné que celle-ci était similaire pour les deux groupes. A l'issue de 6 mois d'élevage, il a été observé des taux de survie de $86,4 \pm 1,5 \%$ et $89,8 \pm 1,1 \%$ respectivement pour la souche Lac Kivu et la souche Nyakabera. En accord avec Diana et al. (1994), les pêches de contrôle organisées régulièrement dans les étangs expérimentaux au cours du cycle d'élevage peuvent avoir été à l'origine des mortalités enregistrées. Dans l'ensemble, les taux de survie observés se situent dans la gamme normale pour l'élevage en étang du tilapia du Nil ( $\geq 75 \%$, d'après Diana et al., 1994). Dans une étude portant sur la comparaison des performances de croissance de 4 souches d'O. niloticus en Egypte, dont une domestique (Abbassa) et trois sauvages (Aswan, Manzalah et Maryut), Abdel-Tawwab (2004) avait noté des valeurs du taux de survie variant de $93.3 \%$ à $100 \%$ pour les souches sauvages et de $96.7 \%$ pour la souche domestique. Cette absence de lien significatif $(p>0,05)$ entre les taux de survie avait été notée par Liti et al. (2005) au Kenya entre les souches d'O. niloticus 'Lac Victoria' (sauvage, 90,40\%), 'Lac Turkana' (sauvage, $84,80 \%$ ) et 'Sagana' (domestique, 87,33\%), après 128 jours de grossissement à partir des juvéniles mâles de 25 à $32 \mathrm{~g}$ nourris avec des feuilles de manioc dans des étangs fertilisés avec de la fumure organique. Avec une fertilisation basée sur du feuillage (Tithonia $s p$ ) et une alimentation supplémentaire constituée d'un mélange de drèche de brasserie, de tourteau palmiste et de farine de fretin, les deux souches étudiées ont réalisé après 6 mois de grossissement, en termes de poids moyen et de gain de poids individuel $285,4 \pm 3,6 \mathrm{~g}$ et respectivement $63 \%$ contre $57 \%$ et $22 \%$ contre $13 \%$. Ce qui dénote une qualité de la récolte relativement meilleure pour la souche Lac Kivu.

$278,0 \pm 4,9 \mathrm{~g}$ pour la souche Lac Kivu, et $199,5 \pm 3,1 \mathrm{~g}$ et $187,1 \pm 5,1 \mathrm{~g}$ pour la souche Nyakabera. Par rapport aux valeurs du poids moyen final, ces résultats sont similaires à ceux obtenus avec 0 . niloticus par Brummet et al. (2004) en station de pisciculture au Cameroun, dans une étude comparative entre une souche fraîchement collectée du Fleuve Sagana et une souche caractérisée par un long passé de domestication. Travaillant avec des juvéniles d'environ $10 \mathrm{~g}$ en sexes mélangés, dans des étangs fertilisés avec un mélange des déchets végétaux et de la bouse de porc, ces auteurs avaient observé après 183 jours d'élevage des poids moyens de $284,3 \mathrm{~g}$ et $178,1 \mathrm{~g}$ respectivement pour la souche Sagana et la souche domestique. Par contre, les poids moyens finals enregistrés par la présente étude pour les deux souches confondues sont inférieurs à ceux obtenus en Côte d'Ivoire (Morissens et al., 1996) avec trois souches d'O. niloticus (dénommées Bouaké, Daloa et Burkina Faso) aux histoires de domestication différentes, élevées en système monosexe mâle avec un aliment en granulés de $30 \%$ de protéines distribué à la demande. En effet, à l'issue d'un cycle de 4 mois seulement les trois souches avaient réalisé des poids moyens variant de $326,86 \mathrm{~g}$ à $384,40 \mathrm{~g}$ à partir des fingerlings d'un poids moyen individuel de $28,55 \mathrm{~g}$ à $39,61 \mathrm{~g}$. Toujours avec O. niloticus, Dan \& Little (2000), dans le Nord du Vietnam, dans une étude comparative de 3 souches domestiques (GIFT, Thaï et Viet) différant à la fois par l'origine et l'histoire de domestication, notent des valeurs de poids moyen final $(199,5 \pm 20,4$ $\mathrm{g} ; 197,0 \pm 22,5 \mathrm{~g} ; 201,3 \pm 21,1 \mathrm{~g})$ globalement comparables à celle observée ici pour la souche Nyakabera (domestique comme elles) mais inférieures à celle de la souche naturelle Lac Kivu. Leur expérimentation portait sur des juvéniles de sexes mélangés d'environ $9 \mathrm{~g}$ élevés en étang pendant 112 jours. Ces différents exemples constituent en somme une illustration de la croissance différentielle des souches d'O. niloticus en fonction non seulement des contextes d'élevage mais aussi de la différence entre souches utilisées, en termes d'origine et d'histoire de domestication. Le gain pondéral moyen quotidien, directement proportionnel au taux de croissance spécifique, est un autre paramètre de croissance qui a 
été examiné dans cette étude. En fonction de la souche utilisée, des conditions d'alimentation et d'élevage, le tilapia peut présenter une croissance individuelle journalière de 2 à $4 \mathrm{~g}$ par jour (Lazard, 2009). Les souches Nyakabera et Lac Kivu ont manifesté une différence tant par la croissance individuelle quotidienne $(1,02 \pm 0,19 \mathrm{~g} / \mathrm{j}$ vs $1,52 \pm 0,16 \mathrm{~g} / \mathrm{j})$ que par le taux de croissance spécifique $(1,52 \pm 0,03 \%$ v vs $1,99 \pm 0,03 \% / j)$, avec une supériorité de cette dernière (Lac Kivu). Les valeurs obtenues pour la croissance individuelle quotidienne dans les deux souches sont en deçà de l'intervalle délimité par Lazard (2009) et de celles observées par Morissens et al. (1996) en Côte d'Ivoire, avec l'élevage monosexe mâle des souches Bouaké (2,8 g/j) et Daloa et Burkina Faso (2,5 g/j pour chacune). Cependant, elles sont supérieures à celles observées au Kenya dans une comparaison de la croissance entre les trois souches locales d'O. niloticus susmentionnées (Liti et al., 2005), dont le gain de poids journalier des juvéniles mâles nourris par du son de blé en étangs fertilisés par de l'engrais inorganique avait varié de $0,8 \mathrm{~g} / \mathrm{j}$ (souches Turkana et Sagana) à $1 \mathrm{~g} / \mathrm{j}$ (souche Victoria). La relative contre-performance des souches locales kenyanes d'O. niloticus relevée ici est surprenante, d'autant plus qu'il est largement reconnu (Dan \& Little, 2000 ; Diagne et al., 2013) que chez les tilapias les populations monosexes mâles ont une croissance plus rapide que les populations élevées en sexes mélangés. A moins qu'on s'en tienne à Capili (1995 in Abdel-Tawwab, 2004) qui avait constaté la relative pauvreté de 3 souches d'origine kenyane à l'issue d'une étude comparative des performances de croissance de 11 souches africaines de tilapias d'origines variées. En outre, les résultats de cette étude sont similaires à ceux de Brummet et al. (2004) qui avaient enregistré un gain de poids journalier de 0.97 $\mathrm{g} / \mathrm{j}$ pour la souche domestique et de 1,55 g/j pour la souche sauvage, les deux ayant été élevées en station à partir des juvéniles en sexes mélangés. Dans la présente étude, le gain de poids individuel observé pour la souche Nyakabera (soit 187,1 g) était en moyenne de $32,7 \%$ inférieur à celui de la souche Lac Kivu (278 g). Ceci est comparable à la valeur (de 39\%) notée dans le même sens par Brummet et al. (2004) lorsqu'ils comparent en station de pisciculture une souche domestique et une souche sauvage d'O. niloticus du Cameroun. Le facteur de condition (K) obtenu dans cette étude pour les deux souches est supérieur à 1 (Tableau 1). Des valeurs de $K$ supérieures à 1 indiquent une bonne condition des poissons (Akongyuure et al., 2015). Cependant, pour ce facteur $(K)$ la valeur observée pour la souche $\mathrm{Lac}$ Kivu $(1,80 \pm 0,02)$ était supérieure $(p<0,0)$ à celle de la souche Nyakabera $(K=1,71 \pm 0,01)$. Sa variation s'est ainsi faite dans le même sens que celle des paramètres de croissance pondérale examinés ci-dessus. L'allure comparée des courbes de croissance pour les souches Nyakabera et Lac Kivu (Figure 1) s'apparente aussi à celle observée en station pour les deux souches camerounaises. Dans les deux cas, la supériorité de la souche sauvage par rapport à la souche domestique tout le long du cycle d'élevage est confirmée. Dans la région du Sud-Kivu, tout comme au Ghana en Afrique de l'Ouest (Ofori et al., 2009) ou à Hanoi en Asie (Cao, 1998), les tilapias de $200 \mathrm{~g}$ et plus sont généralement préférés par les consommateurs. Pour un cycle d'élevage de même durée et dans des conditions d'alimentation similaires, la récolte dans nos étangs expérimentaux avait donné au total $85 \%$ et $70 \%$ des poissons de $200 \mathrm{~g}$ et plus, respectivement pour la souche Lac Kivu et la souche Nyakabera (Figure 2). Une telle différence dans la taille des poissons marchands peut être attribuée, d'après Akongyuure et al. (2015), outre à la variation de la qualité de l'aliment et de l'eau (supposée similaire dans cette expérimentation), à celle des souches en présence. Dans l'ensemble, il ressort de cette étude que la souche Lac Kivu a manifesté une meilleure performance en termes de gain de poids, de taux de croissance spécifique et de facteur de condition comparativement à la souche Nyakabera. Les deux souches d'Oreochromis niloticus étudiées diffèrent par leurs origines, mais en plus la souche Nyakabera se distingue par une longue histoire de domestication. Chez les poissons, la domestication a souvent pour résultat une réduction de la diversité génétique (Osure \& Phelps, 2006) qui se traduit notamment par une baisse des performances de croissance (Bondari et Dunham, 1987; Gjerde, 1988; Galman et al., 1988; Leberg, 1990; Agnèse et al., 1995; Hussain, 2004). Pour O. niloticus en particulier, les études de Eknath et al. (1993), Brummet et al. (2004) et Abdel-Tawwab (2004) ont montré qu'en contexte piscicole les souches à long passé de domestication se montrent moins performantes en termes de croissance que les populations récemment collectées du milieu naturel. La réduction de la variabilité génétique peut être une conséquence de la dépression de consanguinité, résultant elle-même du nombre limité de poissons à l'origine de la souche domestique (Pullin \& Capili, 1988). Ce phénomène pourrait expliquer la relative contre-performance constatée ici pour la souche 
Nyakabera qui à l'origine aurait été fondée à partir d'un stock de géniteurs constitué de 22 femelles et 18 mâles (Pullin, 1988), effectif inférieur au minimum de

\section{RÉFÉRENCES}

Abdel-Tawwab, M., 2004. Comparative study of growth performance and feed utilization of four local strains of Nile tilapia (Oreochromis niloticus L.) collected from different location in Egypt, In: Bolivar R., Mair G. C. and Fitzsimmons K. (eds.). The Proceedings of the 6th International symposium on Tilapia in Aquaculture, Manila, Philippines, pp. 510-517.

Agnèse, J.-F., Otémé, Z. J. \& Gilles, S., 1995. Effects of domestication on genetic variability, fertility, survival and growth rate in a tropical siluriform: Heterobranchus longifilis Valenciennes 1840. Aquaculture, 131, 197-204.

Akongyuure, D. N., Agbeko, E. \& Abarike, E. D., 2015. Preliminary study on growth of mixed sex Nile tilapia (Akosombo strain) in a reservoir-based fish cage in Ghana. Intl J Farm \& Alli Sci., Vol., 4 (1): 13-18.

Amoussou, T. O., Toguyeni, A., Imorou, T. I., Chikou, A. \& Youssao, A. K. I., 2017. Analysis of Morphological Parameters of Wild Populations of Oreochromis niloticus (Linnaeus, 1758) of the Three Hydrographic Basins of Southern Benin. European Journal of Academic Essays, vol. 4, no. 1, 1-11.

Araki, H. \& Schmid, C., 2010. Is hatchery stocking a help or harm? Evidence, limitations and future directions in ecological and genetic surveys. Aquaculture 308, S2-S11.

Bentsen, H. B., Eknath, A. E., Palada-de Vera, M. S., Danting, J. C., Bolivar, H. L., Ruben A. Reyes, R. A., E. Dionisio, E. E., Longalong, F. M., Circa, A. V., Tayamen, M. M. \& Gjerde, B., 1998. Genetic improvement of farmed tilapias: growth performance in a complete diallel cross experiment with eight strains of Oreochromis niloticus. Aquaculture, 160, 145-173.

Bondari, K. \& Dunham, R. A., 1987. Effects of inbreeding on economic traits of channel catfish. Theoretical Applied Genetics, 74: 1-9.

Brummett, R. E., Angoni, D. E. \& Pouomogne, V., 2004. On-farm and on-station comparison of wild and domesticated Cameroonian populations of Oreochromis niloticus. Aquaculture, 242, 157164.
100 à 150 géniteurs recommandé en matière de pisciculture (Smitherman \& Tave, 1987).

Cao, T. B., 1998. Development of pond culture of Thai Nile tilapia (Oreochromis niloticus L.) and its marketability in Hanoi, Vietnam. PhD thesis, Asian Institute of Technology, Bangkok, Thailand, $116 \mathrm{p}$.

Chakraborty, S. B., Mazumdar, D., Chatterji, U. \& Banerjee, S., 2011. Growth of Mixed-Sex and Monosex Nile Tilapia in Different Culture Systems. Turkish Journal of Fisheries and Aquatic Sciences, 11: 131-138.

Dagne, A., Degefu, F. \& Lakew, A., 2013. Comparative Growth Performance of Mono-Sex and MixedSex Nile Tilapia (Oreochromis niloticus L.) in Pond Culture System at Sebeta. Ethiopian. International Journal of Aquaculture, Vol.3, No.7 30-34.

Dan, N. C. \& Little, D. C., 2000. The culture performance of monosex and mixed-sex newseason and overwintered fry in three strains of Nile tilapia (Oreochromis niloticus) in northern Vietnam. Aquaculture, 184, 221-231.

Diana, J. S., Lin, C. K. \& Jaiyen, K., 1994. Supplemental feeding of tilapia in fertilized ponds. Journal of World Aquaculture Society, 25, 497-506.

Eknath, A. E., Tayamen, M. M., Palada-de Vera, M. S., Danting, J. C., Reyes, R. A., Dionisio, E. E., Capili, J. B., Bolivar, H. L., Abella, T. A., Circa, A. V., Bentsen, H. B., Gjerde, B., Gjedrem, T. \& Roger S.V. Pullin, R. S. V., 1993. Genetic improvement of farmed tilapias: the growth performance of eight strains of Oreochromis niloticus tested in different farm environments. Aquaculture, 111, 171-188.

Engle, C. R. \& Neira, I., 2005. Tilapia farm business management and economics: a training manual. Aquaculture CRSP, Oregon State University, Corvallis, $41 \mathrm{p}$.

Galman, O. R., Moreau, J., Hulata, G. \& Avtalion, R. R., 1988. The use of electrophoresis as a technique for the identification and control of tilapia breeding stocks in Israel, In: R.S.V. Pullin, T. Bhukaswan, K. Tonguthai, and J.L. Maclean (Editors), The second ISTA, ICLARM Conf. Proc. 15. Department of Fisheries, 
Bangkok, Thailand, and ICLARM, Manila, Philippines. pp 177-188.

Gjerde, B., 1988. Complete diallele cross between six inbred groups of rainbow trout, Salmo gairdneri. Aquaculture, 75: 71-87.

Hammer, Ø., Harper, D. A. T. \& Ryan, P. D., 2001. PAST: Paleontological Statistics Software Package for Education and Data Analysis. Palaeontologia Electronica, 4(1): $9 \mathrm{p}$.

Hussain, M.G., 2004. Farming of tilapia: Breeding plans, mass seed production and aquaculture techniques. Momin Offset Press, Dhaka, Bangladesh, $149 \mathrm{p}$.

Jayaprakas, V., Tave, D. \& Smitherman, R. O. 1988. Growth of two strains of Oreochromis niloticus and their F1, F2 and backcross hybrids, $p$. 197-201. In R.S.V. Pullin, T. Bhukaswan, K. Tonguthai and J.L. Maclean (eds.) The Second International Symposium on Tilapia in Aquaculture. ICLARM Conference Proceedings 15, 623 p. Department of Fisheries, Bangkok, Thailand, and ICLARM, Manila, Philippines.

Jiwyam, W., 2011. The effect of stocking density on yield, growth, and survival of Asian river catfish (Pangasius bocourti Sauvage, 1880) cultured in cages. Aquacult Int, 19: 987-997.

Khater, A. A. \& Smitherman, R. O. 1988. Cold tolerance and growth of three strains of Oreochromis niloticus, p. 215-218. In R.S.V. Pullin, T. Bhukaswan, K. Tonguthai and J.L. Maclean (eds.) The Second International Symposium on Tilapia in Aquaculture. ICLARM Conference Proceedings 15, 623 p. Department of Fisheries, Bangkok, Thailand, and ICLARM, Manila, Philippines.

Lazard, J. \& Legendre, M., 1996. La reproduction spontanée du tilapia: une chance ou un handicap pour le développement de l'aquaculture africaine ?, p. 82-98. In R.S.V. Pullin, J. Lazard, M. Legendre, J. B. Amon Kothias et D. Pauly (éds.) Le Troisième Symposium International sur le Tilapia en Aquaculture. ICLARM Conf. Proc. 41, 630 p.

Lazard, J., 2009. La pisciculture des tilapias. Cah Agric, vol. 18, n 2-3, 174-182.

Leberg, P. L., 1990. Influence of genetic variability on population growth: implications for conservation. Journal of Fish Biology, 37 (supplement A), 193-195.
Liti, D., Barasa, J. E., Munguti, J. \& Phelps, R., 2005. Evaluation of growth and reproduction capacity of three strains of Nile tilapia, Oreochromis niloticus, found locally in Kenya for use in aquaculture. In: J. Burright, C. Flemming, and H. Egna (Editors), Twenty-Second Annual Technical Report. Aquaculture CRSP, Oregon State University, Corvallis, Oregon, pp. 327336.

Mair, G. C. \& Little, D. C., 1991. Population control in farmed tilapias. NAGA, 14: 8-13.

Mair, G. C., Abucay, J. S., Beardmore, J. A. \& Skibinski, D. O. F., 1995. Growth performance trials of genetically male tilapia (GMT) derived from YY-males in Oreochromis niloticus L.: On station comparisons with mixed sex and sex reversed male populations. Aquaculture 137, 313-322.

McGinnity, P. , Prodohl, P., Ferguson, A., Hynes, R., O' Maoileidigh, N.., Baker, N., Cotter, D., Brendan O'Hea, B., Cooke, D., Rogan, G., Taggart, J. \& Cross, T., 2003. Fitness reduction and potential extinction of wild populations of Atlantic salmon, Salmo salar, as a result of interactions with escaped farm salmon. Proc. R. Soc. Lond. B, 270, 2443-2450.

Morissens, P., Rognon, X. \& Dembélé, I., 1996. Comparaison des performances de croissance et des caractéristiques électrophorétiques de trois souches de Oreochromis niloticus présentes en Côte d'Ivoire, p. 400-407. In R.S.V. Pullin, J. Lazard, M. Legendre, J.B. Amon Kothias et $D$. Pauly (éds.) Le Troisième Symposium International sur le Tilapia en Aquaculture. ICLARM Conf. Proc. 41, 630p.

Nihoreye, F., Muzumani, R. \& Nshombo, M., 2009. Résultats préliminaires sur la différenciation morphologique des deux souches du tilapia du Nil Oreochromis niloticus (Teleostei, Cichlidae) utilisées en pisciculture à l'Est de la R.D. Congo. Cahier du CERUKI, Nouvelle série, 39 : 192-200.

Nihoreye, J. F., Isumbisho, P. M. \& Nyongombe, N. U. (soumis). Caractéristiques de la pisciculture dans la Province du Sud-Kivu (République Démocratique du Congo).

Nihoreye, J. F., Masilya, P. M., Isumbisho, P. M. \& Okitayela, F. O. (soumis). Différenciation morphologique entre deux souches naturelles et une souche cultivée du tilapia du Nil 
Oreochromis niloticus (Linnaeus, 1758) au Sud-Kivu, RD Congo.

Ofori, J. K., Dankwa, H. R., Brummett, R. \& Abban, E. K., 2009. Producing Tilapia in Small Cage in West Africa. WorldFish Center Technical Manual No. 1952. The WorldFish Center, Penang, Malaysia. $16 \mathrm{p}$.

Osure, G. O. \& Phelps, R. P., 2006. Evaluation of reproductive performance and early growth of four strains of Nile tilapia (Oreochromis niloticus, L) with different histories of domestication. Aquaculture, 253 (1-4) : 485494.

Palada-de Vera, M. S. \& Eknath, A. E., 1993. Predictability of individual growth rates in tilapia. Aquaculture, 111: 147-158.

Pechsiri, J. \& Yakupitiyage, A., 2005. A comparative study of growth and feed utilization efficiency of sex-reversed diploid and triploid Nile tilapia, Oreochromis niloticus L. Aquaculture Research, 36: 45-51.

Pullin, R.S.V. (ed.), 1988. Tilapia genetic resources for aquaculture. ICLARM Conf. Proc. 16, $108 \mathrm{p}$.

Pullin, R. S. V. \& Capili, J. B., 1988. Genetic improvement of tilapias: problems and prospects. In: Pullin, R.S.V., Bhukaswan, T., Tonguthayi, K., Maclean, J.L. (Eds.), The Second International Symposium on Tilapia in Aquaculture. ICLARM Conference Proceedings, vol. 15. Department of Fisheries, Bangkok Thailand, and ICLARM, Manila, Philippines, pp. 259-266.
Reisenbichler, R. R., 1988. Relation between Distance Transferred from Natal Stream and Recovery Rate for Hatchery Coho Salmon. North American Journal of Fisheries Management, 8: 2, 172-174.

Ridha, M. T., 2006. Comparative study of growth performance of three strains of Nile tilapia, Oreochromis niloticus L. at two stocking densities. Aquaculture Research, 37, 172-179.

Rognon, X. \& Guyomard, R., 1996. Etude de la variation génétique chez les populations d'élevage de quelques espèces du genre Oreochromis, p. 441-449. In R.S.V. Pullin, J. Lazard, M. Legendre, J.B. Amon Kothias et D. Pauly (éds.) Le Troisième Symposium International sur le Tilapia en Aquaculture. ICLARM Conf. Proc. 41, 630p.

Smitherman, R. O. \& Tave, D., 1987. Maintenance of genetic quality in cultured tilapia. Asian Fisheries Science, 1, 75-82.

Snoeks, L., De Vos, L. \& Thys van den Audenaerde, D.F.E., 1997. The ichthyogeography of Lake Kivu. South African Journal of Science, vol. 93, 579-584.

Tsadik, G. G. \& Bart, A. N., 2007. Characterization and comparison of variations in reproductive performance of Chitralada strain Nile tilapia, Oreochromis niloticus (L.). Aquaculture Research, 38, 1066-1073.

Vrijenhoek, R. C., 1998. Conservation genetics of freshwater fish. Journal of Fish Biology, 53 (supplement A), 394-412. 